Discussiones Mathematicae

\title{
ALMOST SELF-COMPLEMENTARY 3-UNIFORM HYPERGRAPHS
}

\author{
LATA N. KAMBLE \\ Department of Mathematics \\ Abasaheb Garware College \\ Karve Road, Pune-411004 \\ e-mail: lata7429@gmail.com \\ Charusheela M. Deshpande \\ AND \\ Bhagyashree Y. BAM \\ Department of Mathematics \\ College of Engineering Pune \\ Pune-411006 \\ e-mail:dcm.maths@coep.ac.in \\ bpa.maths@coep.ac.in
}

\begin{abstract}
It is known that self-complementary 3-uniform hypergraphs on $n$ vertices exist if and only if $n$ is congruent to 0,1 or 2 modulo 4 . In this paper we define an almost self-complementary 3-uniform hypergraph on $n$ vertices and prove that it exists if and only if $n$ is congruent to 3 modulo 4 . The structure of corresponding complementing permutation is also analyzed. Further, we prove that there does not exist a regular almost self-complementary 3uniform hypergraph on $n$ vertices where $n$ is congruent to 3 modulo 4 , and it is proved that there exist a quasi regular almost self-complementary 3uniform hypergraph on $n$ vertices where $n$ is congruent to 3 modulo 4 .
\end{abstract}

Keywords: uniform hypergraph, self-complementary hypergraph, almost complete 3-uniform hypergraph, almost self-complementary hypergraph, quasi regular hypergraph.

2010 Mathematics Subject Classification: 05C65. 


\section{REFERENCES}

[1] C.R.J. Clapham, Graphs self-complementary in $K_{n}-e$, Discrete Math. 81 (1990) 229-235.

doi:10.1016/0012-365X(90)90062-M

[2] C.J. Colbourn and J.H. Dinitz, The CRC Handbook of Combinatorial Designs (CRC Press, Boca Raton, 1996).

[3] P.K. Das, Almost self-complementary graphs 1, Ars Combin. 31 (1991) 267-276.

[4] P.K. Das and A. Rosa, Halving Steiner triple systems, Discrete Math. 109 (1992) 59-67.

doi:10.1016/0012-365X(92)90278-N

[5] S. Gosselin, Constructing regular self-complementary uniform hypergraphs, Combin. Designs 16 (2011) 439-454.

doi: $10.1002 /$ jcd.20286

[6] A. Hartman, Halving the complete design, Ann. Discrete Math. 34 (1987) 207-224. doi:10.1016/s0304-0208(08)72888-3

[7] L.N. Kamble, C.M. Deshpande and B.Y. Bam, The existence of quasi regular and bi-regular self-complementary 3-uniform hypergraphs, Discuss. Math. Graph Theory 36 (2016) 419-426.

doi:10.7151/dmgt.1862

[8] M. Knor and P. Potočnik, A note on 2-subset-regular self-complementary 3-uniform hypergraphs, Ars Combin. 11 (2013) 33-36.

[9] W. Kocay, Reconstructing graphs as subsumed graphs of hypergraphs, and some selfcomplementary triple systems, Graphs Combin. 8 (1992) 259-276.

doi:10.1007/BF02349963

[10] P. Potočnik and M. Šajana, The existence of regular self-complementary 3-uniform hypergraphs, Discrete Math. 309 (2009) 950-954.

doi:10.1016/j.disc.2008.01.026

[11] G. Ringel, Über Selbstkomplementäre Graphen, Arch. Math. 14 (1963) 354-358. doi:10.1007/BF01234967 
[12] H. Sachs, Über Selbstkomplementäre Graphen, Publ. Math. Debrecen 9 (1962) 270-288.

[13] A. Szymański and A.P. Wojda, A note on $k$-uniform self-complementary hypergraphs of given order, Discuss. Math. Graph Theory 29 (2009) 199-202. doi:10.7151/dmgt.1440

Received 30 September 2015

Revised 23 February 2016

Accepted 3 March 2016 